\title{
Self-assembly of catalytically active colloidal molecules: Tailoring activity through surface chemistry
}

\author{
Rodrigo Soto ${ }^{1,2}$ and Ramin Golestanian ${ }^{2}$ \\ ${ }^{1}$ Departamento de Física, Facultad de Ciencias Físicas y Matemáticas \\ Universidad de Chile, Av. Blanco Encalada 2008, Santiago, Chile \\ ${ }^{2}$ Rudolf Peierls Centre for Theoretical Physics, University of Oxford, Oxford OX1 3NP, UK
}

(Dated: July 31, 2018)

\begin{abstract}
A heterogeneous and dilute suspension of catalytically active colloids is studied as a nonequilibrium analogue of ionic systems, which has the remarkable feature of action-reaction symmetry breaking. Symmetrically coated colloids are found to join up to form self-assembled molecules that could be inert or have spontaneous activity in the form of net translational velocity and spin depending on their symmetry properties and their constituents. The type of activity can be adjusted by changing the surface chemistry and ambient variables that control the surface reactions and the phoretic drift.
\end{abstract}

PACS numbers: $\quad 05.65 .+\mathrm{b}, 47.57 . \mathrm{J}-$, 82.70.Dd

In a solution that maintains a gradient in the concentration of a solute, colloidal particles could be driven up or down the gradient. Since the pioneering work of Derjaguin and coworkers [1, this nonequilibrium transport phenomenon, which is termed as diffusiophoresis, has been studied in detail [2 5]. The gradients that are needed to drive the system away from equilibrium could be generated through catalytic activity, which can be designed to take place asymmetrically on the surface of the colloids themselves, leading to self-propulsion [6 11 and anomalous stochastic dynamics [12. Recent experiments on self-phoretic active colloids have helped characterize their properties and highlight their potential as a versatile means of studying active nonequilibrium systems [1323. Self-diffusiophoresis has been predominantly studied in the context of artificial micro- and nanoscale swimmers, which is currently a very active field of research 24,27.

The non-equilibrium behavior of catalytic colloids is characterized by two independent parameters. First, the surface activity $\alpha$, which describes the production or consumption of chemicals at the colloidal surfaces. For the symmetrically coated colloids we consider here, the concentration profile $C$ is obtained by solving the diffusion coefficient $\nabla^{2} C=0$ with the boundary condition $-\left.D \partial_{r} C\right|_{r=R}=\alpha$, where $R$ is the radius of the colloid and $D$ is the effective diffusivity of the chemicals. The activity results in a concentration profile that decays as $C(r) \sim \alpha R^{2} /(D r)$. The second parameter is the surface mobility $\mu$, which determines how the colloid moves in response to the chemical gradients generated by the colloid itself or by other active colloids in the medium [8, 28. Both parameters can be positive or negative (or zero), independently, and can be in principle controlled by modifying the surface chemistry of the colloids.

A single spherical colloid that is symmetrically coated does not self-propel but phoretic interaction between two such colloidal particles could lead to net motion. (a)
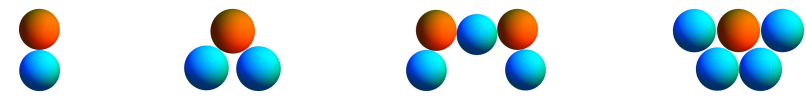

(b)
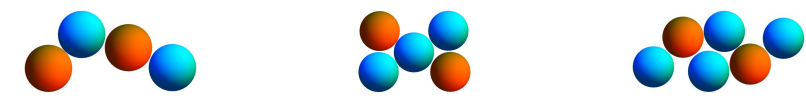

(c)
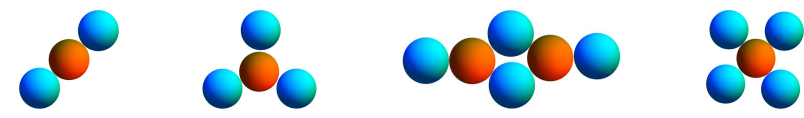

FIG. 1. (color online). Examples of self-assembled active molecules. (a) Self-propelled molecules moving up ( $A$ in orange/dark gray and $B$ in blue/light gray). From left to right: $A B\left(\widetilde{\alpha}_{A}=2, \widetilde{\mu}_{A}=0\right) ; A B_{2}\left(\widetilde{\alpha}_{A}=2, \widetilde{\mu}_{A}=0\right) ; A_{2} B_{3}\left(\widetilde{\alpha}_{A}=2\right.$, $\left.\widetilde{\mu}_{A}=0\right) ; A B_{4}\left(\widetilde{\alpha}_{A}=5, \widetilde{\mu}_{A}=2\right)$. (b) Self-rotating molecules (spinning in the clockwise direction). From left to right: $A_{2} B_{2}$ $\left(\widetilde{\alpha}_{A}=1, \widetilde{\mu}_{A}=3\right) ; A_{2} B_{3}\left(\widetilde{\alpha}_{A}=3, \widetilde{\mu}_{A}=1\right) ; A_{2} B_{4}\left(\widetilde{\alpha}_{A}=2\right.$, $\left.\widetilde{\mu}_{A}=1\right)$. The first one is polar and therefore exhibits spontaneous translation and rotation. The two other cases are nonpolar and therefore they rotate without directed motion. (c) Inert molecules. From left to right: $A B_{2}\left(\widetilde{\alpha}_{A}=1, \widetilde{\mu}_{A}=3\right)$; $A B_{3}\left(\widetilde{\alpha}_{A}=2, \widetilde{\mu}_{A}=1\right) ; A_{2} B_{4}\left(\widetilde{\alpha}_{A}=2, \widetilde{\mu}_{A}=3\right) ; A B_{4}$ $\left(\widetilde{\alpha}_{A}=2, \widetilde{\mu}_{A}=0\right)$.

Within the far-field approximation, the drift velocity of particle 2 due to the activity of particle 1 is given as $\vec{V}_{2}=-\left.\mu_{2} \nabla C\right|_{2} \sim \alpha_{1} \mu_{2} \frac{R^{2} \vec{r}_{12}}{D\left|\vec{r}_{12}\right|^{3}}$, where $\vec{r}_{12}=\vec{r}_{2}-\vec{r}_{1}$, which is different from the drift velocity of particle $1, \vec{V}_{1}=$ $-\left.\mu_{1} \nabla C\right|_{1} \sim-\alpha_{2} \mu_{1} \frac{R^{2} \vec{r}_{12}}{D\left|\vec{r}_{12}\right|^{3}}$. The fact that the actionreaction symmetry is broken implies that the whole pair moves with a velocity proportional to $\left(\alpha_{1} \mu_{2}-\alpha_{2} \mu_{1}\right)$, while the two colloids attract or repel each other with a relative velocity proportional to $\left(\alpha_{1} \mu_{2}+\alpha_{2} \mu_{1}\right)$. Due to this nonequilibrium property, it is possible to vary the parameters such that we obtain stable compounds with various types of activity (see Fig. 1) via self-assembly. This is what we study in this Letter. 
The effective interaction between active colloids is similar to unscreened electrostatic interaction between charged colloidal particles in a fluid. However, we need to generalize the concept of charge to differentiate between the charge that produces the field, $\alpha$, and the charge that responds to the field, $\mu$. The presence of two types of charge gives rise to non-intuitive effects absent in electrostatic systems. For example, if $\alpha_{1}>0$ and $\mu_{1}>0$ for particle 1 , and $\alpha_{2}>0$ while $\mu_{2}<0$ for particle 2, 1 will be repelled by 2 but 2 will be attracted by 1 . We can categorize the different possible scenarios as follows, if only the signs of the two charges $(\alpha, \mu)$ are taken into account. In a mixture of $(++)$ and $(--)$ particles, unequal particles attract and equal particles repel, similar to electrostatics. In a mixture of $(+-)$ and $(-+)$ particles, equal particles attract and unequal particles repel, which is opposite to the electrostatic rules. More complex situations can be obtained by mixing different kind of particles. In such a mixture, we generically have $\alpha_{A} \mu_{B} \neq \alpha_{B} \mu_{A}$, and hence nonequilibrium colloidal activity. Only fine tuning could lead to equilibrium colloidal dynamics for dissimilar colloids (although chemical activity will always be present). Note that a mixture of particles of equal type but different radii also breaks action-reaction symmetry, but exhibits a more restricted phenomenology due to the signs of the charges being fixed. Here, we will concentrate on a $(++)-(--)$ mixture, and adopt the notation of $A=(++)$ and $B=(--)$. This choice allows the selfassembly of different types of particles, and thus, leads to molecules that exhibit activity.

We have performed Brownian dynamics simulation on mixtures of $A$ and $B$ colloids and studied the selfassembly dynamics, and in particular, the spontaneous formation of active molecules. Besides the phoretic interaction, we have incorporated steric forces by treating the colloids as hard spheres all having the same diameter $\sigma$. The addition of the hard core repulsion makes this model similar to the restricted primitive model (RPM) for charged colloids 29], and allows the formation of stable compact colloidal molecules. We have numerically solved the following stochastic equations

$$
\frac{d \vec{r}_{i}}{d t}=\sum_{k \neq i} \frac{\alpha_{k} \mu_{i} R^{2}}{6 \pi D} \frac{\vec{r}_{k i}}{\left|\vec{r}_{k i}\right|^{3}}+\vec{\xi}_{i}(t)=V_{0} \sum_{k} \widetilde{\alpha}_{k} \widetilde{\mu}_{i} \frac{\sigma^{2} \vec{r}_{k i}}{\left|\vec{r}_{k i}\right|^{3}}+\vec{\xi}_{i}(t) .
$$

The first term corresponds to the pair phoretic interactions in the far-field approximation and $\vec{\xi}$ is a random velocity representing white noise of intensity $2 D_{c}$ with $D_{c}$ being the (passive) diffusion coefficient of the colloidal particles, which is proportional to the temperature. Using $\alpha_{0}$ and $\mu_{0}$ as characteristic values of the surface parameters, we have made them dimensionless by defining $\widetilde{\alpha}=\alpha / \alpha_{0}$ and $\widetilde{\mu}=\mu / \mu_{0}$. This gives us the overall velocity scale of $V_{0}=\frac{\alpha_{0} \mu_{0} R^{2}}{6 \pi D \sigma^{2}}=\frac{\alpha_{0} \mu_{0}}{24 \pi D}$.

To simulate the model we adopt a similar strategy as the one described in [30. The system is advanced in time steps $\delta t=0.001 \sigma / V_{0}$. At each time step the drift velocity for each particle is computed summing all pairwise interactions. Then, particles are advanced using a forward Euler scheme that adds the Brownian displacement associated to the time step. At this stage pairs of overlapping particles are reflected, in order, by the same distance they were overlapped. The procedure is repeated until there are no remaining overlaps. This procedure is known to reproduce, in the limit of small time steps, the dynamics of hard core systems [30]. Periodic boundary conditions are used and interactions are treated using the minimal image convention [31, 32]. Although interactions are longranged, for the purpose of this study that focuses on the formation of short scale structures, the Ewald summation scheme was not required. For simplicity, simulations are in $2 \mathrm{D}$, but the interaction is computed considering that solute molecules diffuse in three dimensions, with the effective potential decaying as $1 / r$. This potential, instead of the strictly $2 \mathrm{D} \log r$ form has the advantage that the electrolyte phenomenology is the same as in 3D and there is no global Kosterlitz-Thouless transition.

We define the dimensionless area fraction $\nu=$ $N \pi \sigma^{2} / 4 L^{2}$, where $N$ is the total number of particles and $L$ is the system size, and the dimensionless noise intensity $\widetilde{D}_{c}=D_{c} / V_{0} \sigma$, which we regard as a dimensionless temperature. $\alpha_{0}$ and $\mu_{0}$ are chosen such that for $B$ particles $\widetilde{\alpha}_{B}=\widetilde{\mu}_{B}=-1$. Without loss of generality, we choose $\widetilde{\alpha}_{A} \geq 1$ (the opposite case is obtained by exchanging the role of $A$ and $B$ particles). Since our interest is in the formation of active aggregates composed of a few particles, simulations are performed in the dilute regime $(\nu \ll 1)$. Simulations are performed with $N=1200$ and $L / \sigma=1200-3000$, leading to area fractions in the range $\nu=(1.0-6.5) \times 10^{-4}$. A stationary state (equilibrium or nonequilibrium) can only be reached in the system if there is no net production or consumption of chemicals. This condition requires $N_{A} \alpha_{a}+N_{B} \alpha_{B}=0$, which is equivalent to global neutrality in our electrostatic analogy. Consequently, the relative number of particles is fixed by the relation $N_{B} / N_{A}=\widetilde{\alpha}_{A}$. The initial conditions are such that both species are mixed homogeneously at random. Finally, two particles belong to the same molecule if their relative distance is smaller than $1.5 \sigma$.

At low area fraction and high temperatures, we observe that stable low weight molecules are formed. Some of these molecules are shown in Fig. 1. Depending on the symmetry of the self-assembled molecules, they could exhibit different types of activity, in the form of overall translational and/or rotational self-propulsion. Increasing the area fraction or reducing the temperature leads to the formation of large molecules or clusters. The simplest molecule is $A B$. It has a spontaneous translational velocity in all non-equilibrium conditions. The next molecule that appears is $A B_{2}$, which could acquire two different geometric configurations or isomers: (i) if the molecule 
(a)

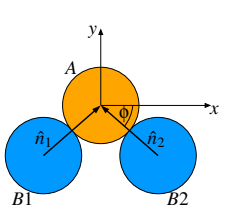

(b)
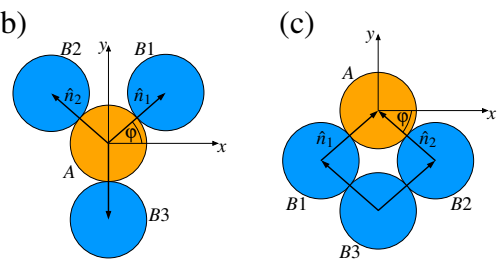

FIG. 2. (color online). Geometric parametrization of the $A B_{2}$ and $A B_{3}$ molecules used in their stability analysis. (a) The angle $\phi$ parameterizes the deviation from a linear chain of the $A B_{2}$ molecule. (b) The angle $\varphi$ parameterizes the deformation of the $A B_{3}$ molecule from the symmetric star configuration. (c) The angle $\varphi$ parameterizes the detachment of one $B$ colloid from the $A B_{3}$ molecule.

is linear, by symmetry, it does not self-propel; (ii) if the molecule adopts a triangular shape it swims steadily. The simplest self-rotating molecule that does not show simultaneous self-propulsion is $A_{2} B_{3}$. Other stable molecules are formed, with varying types of activity (see Fig. 1).

The statistical properties of the $A B$ molecule are obtained by considering the velocities of each colloidal particle [Eq. [1]]. Changing variables to the relative distance $\vec{\rho}=\rho \hat{\rho}$ and the center of mass position $\vec{R}$ (and omitting the noise terms), we obtain for the velocities of the center of mass $\vec{V}_{R}$ an the relative distance $\vec{V}_{\rho}$

$$
\begin{aligned}
& \vec{V}_{R}=V_{0}\left(\widetilde{\mu}_{A}-\widetilde{\alpha}_{A}\right) \frac{\sigma^{2}}{2 \rho^{2}} \hat{\rho}, \\
& \vec{V}_{\rho}=2 U(\rho) \hat{\rho}+V_{0} \sigma^{2}\left(\widetilde{\mu}_{A}+\widetilde{\alpha}_{A}\right) \frac{\hat{\rho}}{\rho^{2}},
\end{aligned}
$$

where $U$ models the hard core repulsion. The propulsion speed of the $A B$ molecule increases as $\rho$ is decreased, achieving the maximum value when the particles are in contact. The corresponding Fokker-Planck equation [when considering the noise terms in Eqs. (2) and (3)] can be simplified by separation of variables since the equation for $\rho$ is completely decoupled, and can be solved at the stationary state to yield the $2 \mathrm{D}$ pair distribution function

$$
P(\rho)= \begin{cases}A \rho e^{\ell / \rho} & ; \rho>\sigma \\ 0 & ; \rho \leq \sigma\end{cases}
$$

where $A$ is a normalization constant. Here, $\ell=$ $V_{0} \sigma^{2}\left(\widetilde{\mu}_{A}+\widetilde{\alpha}_{A}\right) / D_{c}$ is the analogue of the Bjerrum length, which corresponds to the distance at which the distribution function is minimum and marks an energy-entropy crossover. In ionic liquids, this length is used to divide between bound states and free (or ionized) states [29]. We can use $P(\rho)$ to obtain the distribution for the center of mass (propulsion) velocity using Eq. 2, which yields

$$
f\left(V_{R}\right)=\frac{f_{0}}{V_{R}^{2}} \exp \left\{\frac{\ell}{\sigma} \sqrt{\frac{2 V_{R}}{V_{0}\left|\widetilde{\mu}_{A}-\widetilde{\alpha}_{A}\right|}}\right\},
$$

where $f_{0}$ is a normalization constant. The distribution is valid for velocities up to the upper cutoff (maximum

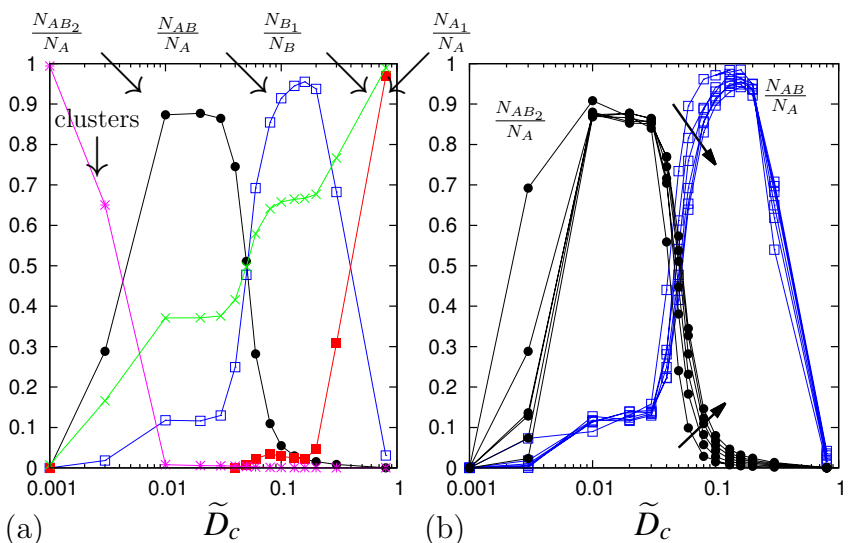

FIG. 3. (color online). Relative populations of different types of molecules as a function of the dimensionless diffusion coefficient (that is proportional to the temperature) in the stationary state. (a) Relative populations for all observed structures for area fraction $\nu=4.36 \times 10^{-4}$. For the clusters, the relative total mass defined as $\sum_{m, n}^{*}(m+n) N_{A_{n} B_{m}} /\left[N_{A}+N_{B}\right]$ is plotted (rather than relative population), where $*$ means that in the sum the following cases are excluded $(n, m)=$ $(1,0),(0,1),(1,1),(1,2)$. (b) The populations of $A B_{2}$ and $A B$ molecules for different area fractions varied in the range $\nu=(1.0-6.5) \times 10^{-4}$, increasing in the direction shown by the arrows. The thin lines are drawn to guide the eye. In the simulations $\widetilde{\alpha}_{A}=3$ and $\widetilde{\mu}_{A}=0$.

value) of $V_{0}\left|\widetilde{\mu}_{A}-\widetilde{\alpha}_{A}\right| / 2$. Our simulations in the studied density range give results in agreement with Eq. (5).

The equilibrium configuration of the $A B_{2}$ molecule, which can exist in two stable states or isomers as mentioned above, can be obtained using a kinematic analysis. Consider a molecule in which a central $A$ colloid is in contact with two $B$ colloids with a bond angle $\phi$ as shown in Fig. 2, The deterministic velocities of the three particles [Eq. (1)] are given by the following expressions

$$
\begin{aligned}
& \vec{V}_{A}=-V_{0} \widetilde{\mu}_{A} \hat{n}_{1}-V_{0} \widetilde{\mu}_{A} \hat{n}_{2}+U \hat{n}_{1}+U \hat{n}_{2}, \\
& \vec{V}_{B 1}=V_{0} \widetilde{\alpha}_{A} \hat{n}_{1}-U \hat{n}_{1}-\frac{V_{0}}{(2 \cos \phi)^{2}} \hat{x}, \\
& \vec{V}_{B 2}=V_{0} \widetilde{\alpha}_{A} \hat{n}_{2}-U \hat{n}_{2}+\frac{V_{0}}{(2 \cos \phi)^{2}} \hat{x},
\end{aligned}
$$

using the same notation to model the hard core repulsion. The unit vectors $\left(\hat{n}_{1}, \hat{n}_{2}\right.$, and $\left.\hat{x}\right)$ are defined in Fig. 2 . Imposing the constraint that the relative $A B$ distances be kept fixed $\left(\vec{V}_{A}-\vec{V}_{B i}\right) \cdot \hat{n}_{i}=0$, the deflection angle satisfies the equation

$$
\dot{\phi}=\frac{2 V_{0} \sin \phi}{\sigma(2-\cos 2 \phi)}\left[\left(\widetilde{\alpha}_{A}-\widetilde{\mu}_{A}\right) \cos \phi-\frac{3}{8 \cos ^{2} \phi}\right] .
$$

The first term in the above equation is due to the activity, whereas the second term is due to the repulsive interaction among the $B$ particles, which favors a linear chain $(\phi=0)$. When $\left(\widetilde{\alpha}_{A}-\widetilde{\mu}_{A}\right)<3 / 8, \phi=0$ is 
stable and the molecule adopts the non-propelling linear isomer configuration. In the opposite case, when $\left(\widetilde{\alpha}_{A}-\widetilde{\mu}_{A}\right)>3 / 8$, any small initial angle is destabilized and the final angle depends on the value of the parameters. In this case the molecule adopts the triangular isomer configuration and swims in the positive $\hat{y}$ direction with a velocity that increases with $\phi$. Replacing the equilibrium angle in Eqs. (6)-(8), yields the swimming velocity $V_{A B_{2}}=V_{0} \sqrt{4\left(\widetilde{\alpha}_{A}-\widetilde{\mu}_{A}\right)^{2}-3^{2 / 3}\left(\widetilde{\alpha}_{A}-\widetilde{\mu}_{A}\right)^{4 / 3}} / 3$. One could induce a transition between the isomers and modify the molecule activity by changing the values of the charges, which depend on the temperature and other ambient variables.

A similar analysis for $A B_{3}$ molecule, when $\widetilde{\alpha}_{A}=3$ and $\widetilde{\mu}_{A}=0$, reveals that it is unstable, and that it will break up into an $A B_{2}$ molecule and a free $B$ colloid, through a detachment process characterized by the steady increase of the angle $\varphi$ as shown in Figs. $2 \mathrm{p}$ and 2 . In the case of $\widetilde{\alpha}_{A}=4$ and $\widetilde{\mu}_{A}=2$, it is possible to have stable self-propelling $A B_{3}$ molecules, although they fluctuate strongly and the swimming direction changes rapidly. A movie of both possibilities is shown in the Supplemental Material 33.

It is interesting to note that despite the condition of global neutrality and in contrast with what we expect from ionic bonds, small stable molecules are not necessarily neutral. Moreover, neutral molecules could even be unstable as it is the case for $A B_{3}$. One can argue that although non-neutral molecules attract monomers and other molecules, when they are active they remain charged because their propulsion strongly hinders the capture process and by the detachment of colloids, as can be observed in the Supplemental Material movies [33.

The stationary distribution of molecules is studied for $\widetilde{\alpha}_{A}=3$ and $\widetilde{\mu}_{A}=0$, in which case the $A B$ an $A B_{2}$ molecules are active. The number of these molecules is presented in Fig. 3 for simulations done with $N=1200$ and various densities and temperatures. We observe that in the steady state the system forms small molecules with the overwhelming majority being of the form $A B_{n}$ (>98\% for $n \leq 4)$ and no large clusters, except for very low temperatures $\left(\widetilde{D}_{c} \lesssim 0.01\right)$ as shown in Fig. 3a. The types of self-assembled molecules are predominantly controlled by temperature, with little dependence on density in the range studied as Fig. 3p shows. Between $\widetilde{D}_{c}=0.015$ and $\widetilde{D}_{c}=0.05$ there is a sharp transition from $A B_{2}$ to $A B$. This observation is generic and not specific to this particular case. For example, in the case of $\widetilde{\mu}_{A}=1$, a similar behavior is obtained but the transition temperature is located between $\widetilde{D}_{c}=0.05$ and $\widetilde{D}_{c}=0.15$.

Note that although the relative concentration of molecules as a function of temperature follows a similar trend as in equilibrium, contrary to the equilibrium case the stationary distributions cannot be computed in terms of effective molecular free energies or equilibrium constants. If this were the case, the relative concentrations would satisfy $N_{A B_{2}}=K N_{B_{1}} N_{A B}$ at low densities, with $K$ the equilibrium constant being a function of the temperature only. This and similar relations for the other molecules, however, are not quantitatively satisfied here.

We have studied a mixture of active colloids with asymmetric mutual diffusiophoretic interactions and shown that they self-assemble into active molecules. Moreover, we have found that the self-assembled structures are stable (distinct like molecules that form from ions of given valence), exhibit novel non-equilibrium dynamics, and present different types of activity that can be varied by changing simple parameters. To capture the main features of the self-assembled molecules we simplified the colloidal interaction by only considering the far-field contribution (effective potential decaying as $1 / r$ ). To make quantitative predictions, corrections at short distances should be included to consider the exact solution of the diffusion equation with the appropriate boundary conditions, and the depletion of chemicals near the colloidal surfaces. However, we expect that these corrections will only modify the result quantitatively, and that the main qualitative features of the above phenomenology would remain the same. Hydrodynamic interactions between colloids have not been considered. Since they respect the action-reaction symmetry, we do not expect them to introduce qualitative changes in our description except for the suppression of relative slip between colloids due to the lubrication layer and possible quantitative changes in the value of the stationary equilibrium angle in small molecules. For significantly larger clusters, however, the effect of hydrodynamic interactions could be more complicated, and warrants further investigations. Allowing for particles to move in three dimensions does not change the global picture presented here. Geometric modifications will alter the thresholds for the transitions between isomers and new isomer configurations (e.g., a pyramid for $A B_{3}$ ) will emerge. However, we expect no new phenomenology to emerge due to the possibility of bending in the third direction, except for the formation of active chiral molecules. We relegate a detailed examination of the above effects to a future publication.

This research is supported by Fondecyt Grant No. 1100100 and Anillo grant ACT 127 (R.S.), and Human Frontier Science Program (HFSP) grant RGP0061/2013 (R.G.).

[1] B.V. Derjaguin, G.P. Sidorenkov, E.A. Zubashchenkov, and E.V. Kiseleva, Kolloidn. Zh. 9, 335 (1947).

[2] J.L. Anderson, Ann. Rev. Fluid Mech. 21, 61 (1989).

[3] F. Jülicher and J. Prost, Eur. Phys. J. E 2927 (2009).

[4] B. Abecassis et al., Nature Mat. 7785 (2008).

[5] J. Palacci, B. Abecassis, C. Cottin-Bizonne, C. Ybert, 
and L. Bocquet, Phys. Rev. Lett. 104, 138302 (2010).

[6] R. Golestanian, T.B. Liverpool, and A. Ajdari, Phys. Rev. Lett. 94, 220801 (2005).

[7] G. Rückner and R. Kapral, Phys. Rev. Lett. 98, 150603 (2007).

[8] R. Golestanian, T.B. Liverpool, and A. Ajdari, New J. Phys. 9, 126 (2007).

[9] M.N. Popescu, M. Tasinkevych and S. Dietrich, Europhys. Lett. 9528004 (2011).

[10] B. Sabass and U. Seifert, Phys. Rev. Lett. 105, 218103 (2010).

[11] S. Michelin, E. Lauga, and D. Bartolo, Phys. Fluids 25, 061701 (2013).

[12] R. Golestanian, Phys. Rev. Lett. 102, 188305 (2009).

[13] J.R. Howse et al., Phys. Rev. Lett. 99, 048102 (2007).

[14] H. Ke, S. Ye, R.L. Carroll, and K. Showalter, J. Phys. Chem. A 114, 5462 (2010).

[15] L.F. Valadares, Y.-G. Tao, N. S. Zacharia, V. Kitaev, F. Galembeck, R. Kapral, and G. A. Ozin, Small 7, 565 (2010).

[16] S. Ebbens, R. A. L. Jones, A. J. Ryan, R. Golestanian, and J. R. Howse, Phys. Rev. E 82, 015304 (R) (2010).

[17] J. Palacci, C. Cottin-Bizonne, C. Ybert, and L. Bocquet, Phys. Rev. Lett. 105, 088304 (2010).

[18] G. Volpe, I. Buttinoni, D. Vogt, H.-J. Kümmerer, and C. Bechinger, Soft Matter 7, 8810 (2011).

[19] S. Ebbens and J.R. Howse, Langmuir 27, 12293 (2011).

[20] S. Ebbens, M.-H. Tu, J. R. Howse, and R. Golestanian, Phys. Rev. E 85, 020401 (R) (2012).
[21] F. Kümmel, B. ten Hagen, R. Wittkowski, I. Buttinoni, R. Eichhorn, G. Volpe, H. Löwen, and C. Bechinger, Phys. Rev. Lett. 110, 198302 (2013).

[22] J. Palacci, S. Sacanna, A. Vatchinsky, P.M. Chaikin, and D.J. Pine, J. Am. Chem. Soc., 135, 15978 (2013).

[23] R. Kapral, J. Chem. Phys. 138, 020901 (2013).

[24] W. F. Paxton et al., Angew. Chem. Int. Ed. 45, 5420 (2006).

[25] E. R. Kay, D. A. Leigh, and F. Zerbetto, Angew. Chem. Int. Ed. 46, 72 (2007).

[26] A. Sen, M. Ibele, Y. Hong, and D. Velegol, Faraday Discuss. 143, 15 (2009).

[27] S. Sengupta, M.E. Ibele, and A. Sen, Angew. Chem. Int. Ed. 51, 8434 (2012).

[28] Although we have used the language of diffusiophoresis throughout the Letter, the scheme presented here is generic for all phoretic transport mechanisms, as discussed in Ref. 8].

[29] Y. Levin, Rep. Prog. Phys. 65, 1577 (2002).

[30] P. Strating, Phys. Rev. E 59, 2175 (1999).

[31] M. P. Allen and D. J. Tildesley, Computer Simulations of Liquids (Oxford Science, New York, 1987).

[32] D. Frenkel and B. Smit, Understanding Molecular Simulations. From Algorithms to Applications (Academic Press, Boston, 2002), second edition.

[33] See Supplemental Material at http://link.aps.org/supplemental/XXXXX for videos of the transient dynamics and the active molecular dynamics. 